Commentary for Target Article: "Is Coding a Relevant Metaphor for the Brain?" (Romain Brette)

Article Word Counts: Abstract (58), Main text (971), References (283), Entire text (1378).

Institutional Mailing Address: Av. Eduard Maristany 10-14, Edifici C, 08019 Barcelona, Spain.

Institutional Telephone Number: +34 934011918

Author Emails: x.d.arsiwalla@gmail.com (Correspondence email)

ruben.moreno@upf.edu pverschure@ibecbarcelona.eu

\title{
Beyond Neural Coding? Lessons from Perceptual Control Theory
}

\author{
Xerxes D. Arsiwalla ${ }^{1, *}$, Ruben Moreno Bote ${ }^{3,4}$, Paul Verschure ${ }^{1,2}$ \\ ${ }^{1}$ Institute for Bioengineering of Catalonia \& Barcelona Institute for Science and Technology. \\ ${ }^{2}$ Catalan Institute for Advanced Studies, Barcelona, Spain. \\ ${ }^{3}$ Center for Brain and Cognition, Department of Information and Communication Technologies, \\ Universitat Pompeu Fabra, Barcelona, Spain. \\ ${ }^{4}$ Serra Húnter Fellow Programme, Universitat Pompeu Fabra, Barcelona, Spain. \\ *Email: x.d.arsiwalla@gmail.com
}

\begin{abstract}
$\underline{\text { Abstract }}$
Pointing to similarities between challenges encountered in today's neural coding to $20^{\text {th }}$ century behaviorism, we draw attention to lessons learnt from resolving the latter. In particular, Perceptual Control Theory posits behavior as a closedloop control process with immediate and teleological causes. With two examples, we illustrate how these ideas may also address challenges facing current neural coding paradigms.
\end{abstract}

\section{$\underline{\text { Main Text }}$}

It is noteworthy that many of the challenges to today's neural coding paradigms, pointed out in Brette (2019), are strikingly similar to problems encountered with $20^{\text {th }}$ century behaviorism. Other authors have also alluded to this correspondence (Gomez-Marin,2017; Fiorillo et al.,2014). Gomez-Marin comments that once it became possible to look inside neural tissue, the philosophical essence of behaviorism made its way back. Behavior was once 
again relegated to linear responses, but this time to internal causes. According to Powers (1973), behavior is control of the animal, by the animal and should be studied as a circular process from the perspective of the animal, including both, immediate as well as teleological causes. In other words, once one knows the 'inside', would one really know everything on the 'outside'? The issue seems to be with what is meant by causation in these paradigms. Admittedly, both behaviorism as well as coding do not consider circular causation, nor do they address teleological aspects of causation. Circular causation also features prominently in the 'enactivist' philosophy of mind, where an organism's action and perception are constantly shaped by mutual interaction with its environment (Varela et al.,1991; Verschure et al.,2003). The other problem seems to be the way information theory is used. Fiorillo et al. make the case for a shift in perspective, where information conditioned on the neuron's biophysics, rather than the experimenter's knowledge, does away with the need for a 'neural code' (arguing that encoding/decoding only make sense from the perspective of an external observer). These works substantiate Brette's main argument, claiming that the implicit definition of 'code' used in neural paradigms does not encompass aspects of causation or representation relevant for bridging brain and behavior.

However, there are lessons we can learn from behaviorism. More specifically, solutions to those problems might also prove insightful today for addressing difficulties encountered in current neuroscience paradigms. In particular, Perceptual Control Theory (PCT), championed by William Powers (1973) was one such response to behaviorism. PCT originated from early cybernetics, which was concerned with control and autonomy in living organisms. PCT posits that behavior is the process of closed-loop control of what the animal senses, rather than a linear causal response to stimuli. The main insight of PCT was that autonomous goal-directed behavior necessitates a hierarchical control architecture, where higher-level controllers are coupled to lower-level controllers such that the output of one layer provides a reference to the next. An organism performs actions to cancel the effects of disturbances in what it senses, in order to achieve intended perceptual consequences. Reference signals across this hierarchy constitute immediate or distal goals. The specification of goals for achieving intended consequences constitute purpose. Ultimate purposes are assumed to be intrinsic, tied to survival drives.

How does this link to neural coding? The important point is that neural coding theories are also trying to explain behavior. However, they attempt to do so by anchoring on linear input-output neural mechanisms, akin to a 'switchboard' model of behavior (Powers). The alternative is that neural activity influences actions and actions influence neural activity in terms of what the animal perceives. Behavior is thus a circular control process. This solicits an explanation of how and why the animal itself controls the 'switchboard'. If neural coding approaches ultimately seek to explain perception and action, then ideas from PCT suggest ways to progress beyond metaphors. Namely, PCT calls for a 
process-theoretic view of the brain-body-environment system, where circular causation is implemented through hierarchical feedback control.

How does control theory address challenges that neural coding theories face? For this, we now turn to control architectures built upon a hierarchy of forward models. These offer a viable solution to closed-loop adaptive and anticipatory processes. The forward models we refer to are internal models acquired during learning and development. These are akin to the physics and psychology engines discussed in Lake et al.,2017. Let us point to two specific examples, where systems-level control architectures with forward models offer the type of closed-loop causal explanations mentioned above. The first example comes from cerebellar motor control. Herreros et al.,2016 and Maffei et al.,2017 have proposed an anticipatory control scheme involving the vestibular system, where the cerebellum implements a forward model of the motor system being controlled. The model generates anticipatory adjustments to counteract postural and equilibrium disturbances during voluntary movements. It does so by learning to anticipate counterfactual errors in motor action given sensory stimuli and an internal model of the motor system. This closed-loop control architecture has been proposed to model eye-blink conditioning, vestibulo-ocular reflexes and visual tracking, all involving cerebellar circuits. Physiologically, this implies that timing-dependent plasticity rules of Purkinje cell synapses implement a model of the motor system being controlled by that cerebellar microcircuit (Suvrathan et al.,2016). Our second example refers to the hierarchical mirror system identified in the brain (Gazzola \& Keysers, 2009). This extends across several brain regions including the motor, somatosensory and gustatory areas. These systems have been shown to hierarchically implement internal forward and inverse models relating to one's own sensations. The mirror system projects outputs of these self-models upon others during social interactions. This hierarchy of forward and inverse models has been used to explain empathy, somatic sensations in others and emotions in social cognition (Keysers et al.,2010). Both these examples illustrate the role of control and internal models in brain and behavior.

In closing, one would agree that encoding or decoding of experimental variables in behavioral paradigms are valuable epistemological constructs for the experimenter. If 'goals' and 'purposes' are necessary to describe behavior emerging from dynamical systems engaged in hierarchical control (Powers), then neuronal coding protocols might be useful tools to identify precisely those variables that define the underlying closed-loop dynamical system. However, it is also true that one ought to refrain from the fallacy of extending conditional epistemic descriptors to ontological explanations of brain and behavior.

\section{References}

Brette, R. (2019). Is coding a relevant metaphor for the brain? Behavioral and Brain Sciences, $1-44$. 
Fiorillo, C. D., Kim, J. K., \& Hong, S. Z. (2014). The meaning of spikes from the neuron's point of view: predictive homeostasis generates the appearance of randomness. Frontiers in

Computational Neuroscience, 8, 49.

Gazzola, V., \& Keysers, C. (2009). The observation and execution of actions share motor and somatosensory voxels in all tested subjects: single-subject analyses of unsmoothed fMRI data. Cerebral Cortex, 19(6), 1239-1255.

Gomez-Marin, A. (2017). Causal circuit explanations of behavior: Are necessity and sufficiency necessary and sufficient? In Decoding Neural Circuit Structure and Function (pp. 283-306), Springer.

Herreros, I., Arsiwalla, X. D., \& Verschure, P. (2016). A forward model at Purkinje cell synapses facilitates cerebellar anticipatory control. In Advances in Neural Information Processing Systems (pp. 3828-3836).

Keysers, C., Kaas, J. H., \& Gazzola, V. (2010). Somatosensation in social perception. Nature Reviews Neuroscience, 11(6), 417.

Lake, B. M., Ullman, T. D., Tenenbaum, J. B., \& Gershman, S. J. (2017). Building machines that learn and think like people. Behavioral and Brain Sciences, 40.

Maffei, G., Herreros, I., Sanchez-Fibla, M., Friston, K. J., \& Verschure, P. F. (2017). The perceptual shaping of anticipatory actions. Proceedings of the Royal Society B: Biological Sciences, 284(1869), 20171780.

Powers, W. T. (1973). Feedback: Beyond Behaviorism. Science, 179(4071), 351-356.

Suvrathan, A., Payne, H. L., and Raymond, J. L. (2016). Timing rules for synaptic plasticity matched to behavioral function. Neuron, 92(5):959-967.

Varela, F., Thompson, E., \& Rosch, E. (1991). The Embodied Mind: Cognitive Science and Human Experience. MIT Press.

Verschure, P. F., Voegtlin, T., \& Douglas, R. J. (2003). Environmentally mediated synergy between perception and behaviour in mobile robots. Nature, 425(6958), 620. 